\title{
Photooxidations of
}

\section{2-( $\gamma, \varepsilon$-dihydroxyalkyl) furans in \\ Water: Synthesis of DE-Bicycles of the Pectenotoxins}

\author{
Antonia Kouridaki, Tamsyn Montagnon, Maria Tofi and Georgios Vassilikogiannakis* \\ Department of Chemistry, University of Crete, Vasilika Vouton, 71003 Iraklion, Crete, \\ Greece \\ vasil@chemistry.uoc.gr
}

Received Date (will be automatically inserted after manuscript is accepted)

\section{ABSTRACT}

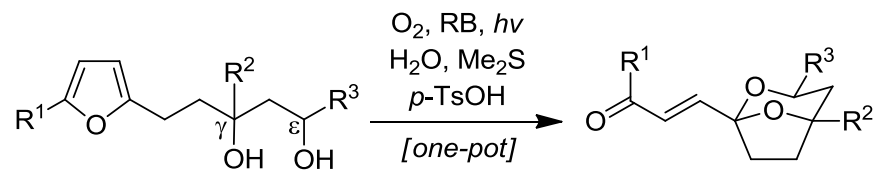

Photooxygenations of 2-( $\gamma, \varepsilon$-dihydroxyalkyl) furans in $\mathrm{H}_{2} \mathrm{O}$ followed by in situ reduction and ketalization affords, in one synthetic operation, DE-bicyclic ketals of the pectenotoxins.

Polyoxygenated molecules are ubiquitous as targets in synthesis. Currently, a high proportion of the most highlyprized and sought-after natural products and bioactive molecules fall into this category. However, it is also a category that lags behind in developmental terms when evaluated by the "ideal synthesis" criteria. ${ }^{1}$ Strategies for the construction of polyoxygenated motifs are often executed in a stepwise fashion; are rife with wasteful redox-shuttling (frequently using environmental unsound and/or atom inefficient reagents); and, are an intransigent last bastion for the overuse of protecting groups. It is a primary goal of our research to continue to contribute novel methods for the synthesis of a wide range of polyoxygenated motifs ${ }^{2}$ using a new, sustainable, and green approach to the problem-in-hand; in which, singlet oxygen $\left({ }^{1} \mathrm{O}_{2}\right)$ plays a pivotal role. Herein, we report one such new method which may be directed towards the

${ }^{1}$ (a) Young, I. S.; Baran, P. S. Nat. Chem. 2009, 1, 193. (b) Gaich, T.; Baran, P. S. J. Org. Chem. 2010, 75, 4657. (c) Newhouse, T.; Baran, P. S.; Hoffmann, R. W. Chem. Soc. Rev. 2009, 38, 3010.

${ }_{2}$ (a) Montagnon, T.; Tofi, M.; Vassilikogiannakis, G. Acc. Chem. Res. 2008, 41, 1001. (b) Montagnon, T.; Noutsias, D.; Alexopoulou, I.; Tofi, M.; Vassilikogiannakis, G. Org. Biomol. Chem. 2011, 9, 2031.

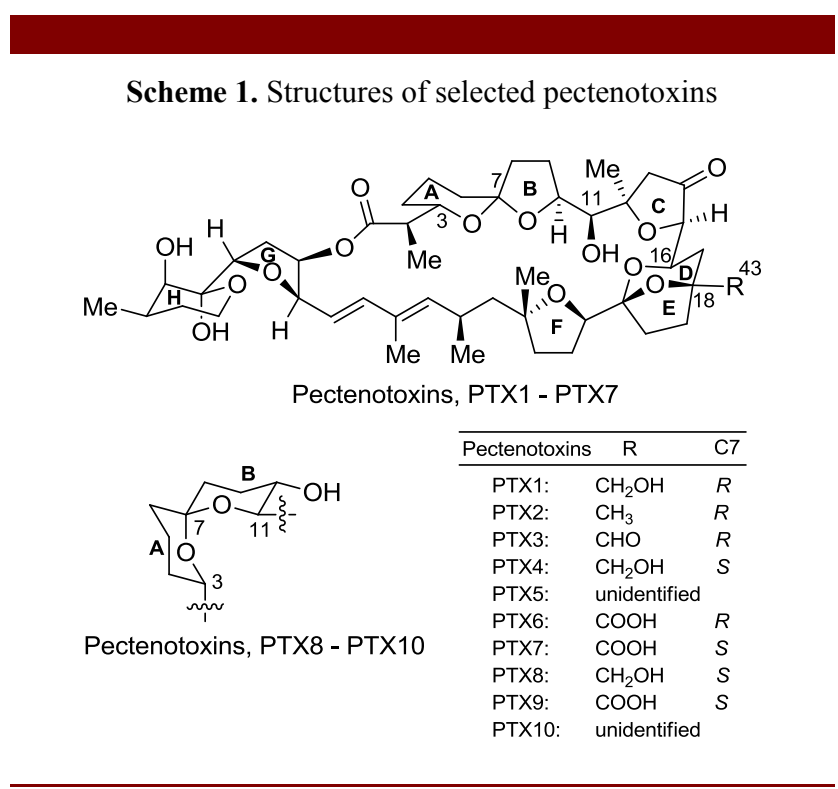


synthesis of the DE-bicycle ${ }^{3}$ of various different pectenotoxins (Scheme 1). Crucially, as will be fully delineated later, these analogues exhibit critical variations in substitution (at the pectenotoxin $\mathrm{C}-18$ ) of the DEbicycle which posed no threat to the method's implementation, but which may hinder other approaches. The described method also represents a key step forward, as the regular use of water, as solvent, for the central cascade reaction sequence, is shown to be highly effective, thus making the attainment of an "ideal green synthesis" an evermore realistic prospect. ${ }^{4}$

The pectenotoxins (PTXs) ${ }^{5}$ have excited much synthetic interest $^{6,7}$ due to their perceived potential as useful cytotoxic agents $^{5 b}$ (especially because they act via a novel mode of $\mathrm{F}$-actin disruption ${ }^{8}$ ) coupled with their low natural abundance. Recently, the groups of Pihko, ${ }^{9}$ Brimble $^{10}$ and Micalizio ${ }^{11}$ have published their own approaches to fragments which include the DE-ring ${ }^{3}$ unit, building on an earlier example from the group of Roush. ${ }^{12}$ The groups of Paquette ${ }^{13}$ and Fujiwara ${ }^{14}$ have completed

\footnotetext{
${ }^{3}$ There is some deviation in the assignment of E-ring in the published pectenotoxin literature. When we refer to the E-ring throughout this manuscript we are refering to the E-ring as it is depicted in Figure 1.

${ }^{4}$ Noutsias, D.; Alexopouou, I.; Montagnon, T.; Vassilikogiannakis, G. Green Chem. 2012, 14, 601 .

${ }^{5}$ For isolation see: (a) Yasumoto, T.; Murate, M.; Oshima, Y.; Sano, M.; Matsumoto, G. K.; Clardy, J. Tetrahedron 1985, 41, 1019. (b) Sasaki, K.; Wright, J. L. C.; Yasumoto, T. J. Org. Chem. 1998, 63, 2475. (c) Daiguji, M.; Satake, M.; James, K. J.; Bishop, A.; Mackenzie, L.; Naoki, H.; Yasumoto, T. Chem. Lett. 1998, 7, 653. (d) Wilkins, A. L.; Rehmann, N.; Torgersen, T. R.; Keogh, M.; Petersen, D.; Hess, P.; Rise, F.; Miles, C. O. J. Agric. Food. Chem. 2006, 54, 5672. (e) Miles, C. O.; Wilkins, A. L.; Hawkes, A. D.; Jensen, D. J.; Selwood, A. I.; Beuzenberg, V.; MacKenzie, A. L.; Cooney, J. M.; Holland, P. T. Toxicon 2006, 48, 152 and references therein.

${ }^{6}$ For total syntheses of PTX4 and PTX8, see: (a) Evans, D. A.; Rajapakse, H. A.; Stenkamp, D. Angew. Chem., Int. Ed. 2002, 41, 4569. (b) Evans, D. A.; Rajapakse, H. A.; Chiu, A.; Stenkamp, D. Angew. Chem. Int. Ed. 2002, 41, 4573.

${ }^{7}$ For a review covering PTX synthetic studies up to 2006, see: Halim, R.; Brimble, M. A. Org. Biomol. Chem. 2006, 4, 4048. For later studies excluding DE-bicycle related work which is covered in refs 9-14, see; (a) Kolakowski, R. V.; Williams, L. J. Tetrahedron Lett. 2007, 48, 4761. (b) Vellucci, D.; Rychnovsky, S. D. Org. Lett. 2007, 9, 711. (c) Lotesta, S. D.; Hou, Y.; Williams, L. J. Org. Lett. 2007, 9, 869. (d) Heapy, A. M.; Wagner, T. W.; Brimble, M. A.Synlett 2007, 2359. (e) Heapy, A. M.; Brimble, M. A. Tetrahedron 2010, 66, 5424. (f) Joyasawal, S.; Lotesta, S. D.; Akhmedov, N. G.; Williams, L. J. Org. Lett. 2010, 12, 988. (g) Aho, J. E.; Piisola, A.; Krishnan, K. S.; Pihko, P. M. Eur. J. Org. Chem. 2011, 1682. (h) Vassilikogiannakis, G.; Alexopoulou, I.; Tofi, M.; Montagnon, T. Chem. Commun. 2011, 47, 259. (i) Kemppainen, E. K.; Sahoo, G.; Valkonen, A.; Pihko, P. M. Org. Lett. 2012, 14, 1086.
}

${ }^{8}$ (a) Chae, H.-D.; Choi, T.-S.; Kim, B.-M.; Hung, J. H.; Bang, Y.-J.; Shin, D. Y. Oncogene 2005, 24, 4813. (b) Shin, D. Y.; Kim, G. Y.; Kim, N. D.; Jung, J. H.; Kim, S. K.; Hang, H. S.; Choi, Y. H. Oncol. Rep. 2008, 19, 517.

${ }^{9}$ Helmboldt, H.; Aho, J. E.; Pihko, P. M. Org. Lett. 2008, 10, 4183. (b) Aho, J. E.; Salomäki, E.; Rissanen, K.; Pihko, P. M. Org. Lett. 2008, 10, 4179 .

${ }^{10}$ Carley, S.; Brimble, M. A. Org. Lett. 2009, 11, 563

${ }_{11}^{11}$ Canterbury, D. P.; Micalizio, G. C. Org. Lett. 2011, 13, 2384.

${ }_{12}$ Micalizio, G. C.; Roush, W. R. Org. Lett. 2001, 3, 1949.

13 (a) O'Connor, P. D.; Knight, C. K.; Friedrich, D.; Peng, X.; Paquette, L. A. J. Org. Chem. 2007, 72, 1747. (b) Bondar, D.; Liu, J.; Müller, T.; Paquette, L. A. Org. Lett. 2005, 7, 1813.

14 (a) Fujiwara, K.; Aki, Y.; Yamamoto, F.; Kawamura, M.; Kobayashi, M.; Okano, A.; Awakura, D.; Shiga, S.; Murai, A.; Kawai, H.; Suzuki, T. Tetrahedron Lett. 2007, 48, 4523. (b) Fujiwara, K.; Suzuki, Y.; Koseki, N.; Murata, S.-I.; Murai, A.; Kawai, H.; Suzuki, T. Tetrahedron Lett. 2011, 52, 5589.

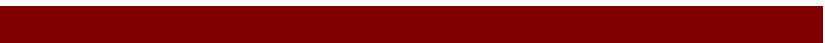

Scheme 2. The concept
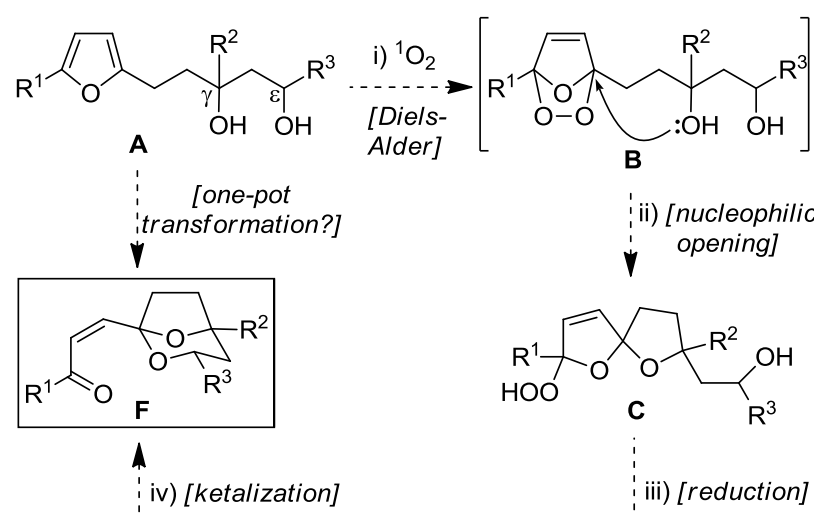

ii) [nucleophilic

$\dot{v}$ opening]
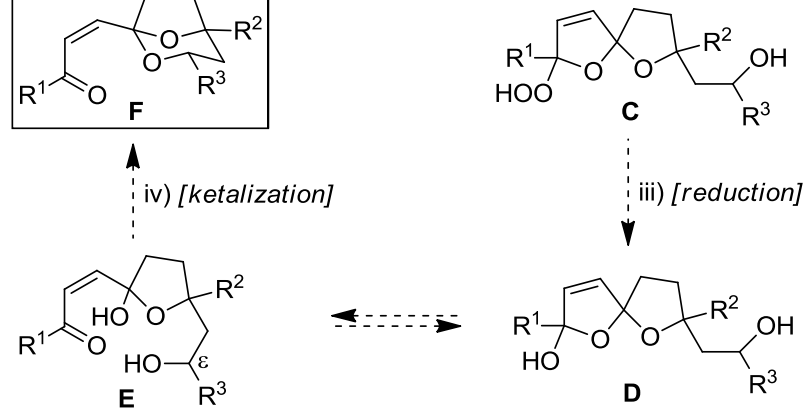

PTX sections which include the necessary, but, as yet, uncyclised, backbone of the DE-bicycle section.

The outline of the unique and simple one-pot approach, by which we hoped to access with minimal wastage and using maximum step- and atom-economies, the DE-ring fragments of the many different pectenotoxins, is shown in Scheme 2. Previously, we had reported a "super-cascade" reaction sequence, mediated by singlet oxygen, to synthesise very rapidly from a readily accessible difuran precursor the ABC-ring framework of the pectenotoxins, ${ }^{\text {Th }}$ so it was envisaged that this new piece of work would contribute to a bigger overall plan in which singlet oxygen-mediated cascade reactions might be used to construct all the key fragments that go to making up the pectenotoxins very effectively and in short synthetic sequences.

Thus, it was hoped that following the cascade-initiating reaction wherein singlet oxygen adds to a furan, ${ }^{15}$ bearing an appendage with both $\gamma$ - and $\varepsilon$-hydroxyl groups, in a [4+2]-addition manner $(\mathbf{A} \rightarrow \mathbf{B}$, Scheme 2), the resultant ozonide would be opened by an intramolecular nucleophilic attack of the $\gamma$-hydroxyl $(\mathbf{B} \rightarrow \mathbf{C})$ reminiscent of those we have already envoked to synthesise various spiroketals. ${ }^{\text {7h, } 16}$ In situ reduction of the peroxide functionality should then afford spiroketal $\mathbf{D}$, which it was hoped would collapse to afford enone $\mathbf{E}$. As the culmination of this ambitious cascade reaction sequence, it was hoped that a ketalisation event, involving the $\varepsilon^{-}$ hydroxyl, would then occur trapping out hemiketal $\mathbf{E}$ to furnish the desired DE-bicycle $(\mathbf{E} \rightarrow \mathbf{F})$. It is important to

15 (a) Foote, C. S.; Wuesthoff, M. T.; Wexler, S.; Burstain, I. G.; Denny, R.; Schenck, G. O.; Schulte-Elte, K. H. Tetrahedron 1967, 23 , 2583. (b) Gollnick, K.; Griesbeck, A. Tetrahedron 1985, 41, 2057. (c) Feringa, B. L. Recl. Trav. Chim. Pays-Bas 1987, 106, 469.

${ }^{16}$ Georgiou, T.; Tofi, M.; Montagnon, T.; Vassilikogiannakis, G, Org. Lett. 2006, 8, 1945. (b) Tofi, M.; Montagnon, T.; Georgiou, T.; Vassilikogiannakis, G. Org. Biomol. Chem., 2007, 5, 772. 
Scheme 3. Photooxidation of 2-( $\gamma, \varepsilon$-dihydroxyalkyl) furans
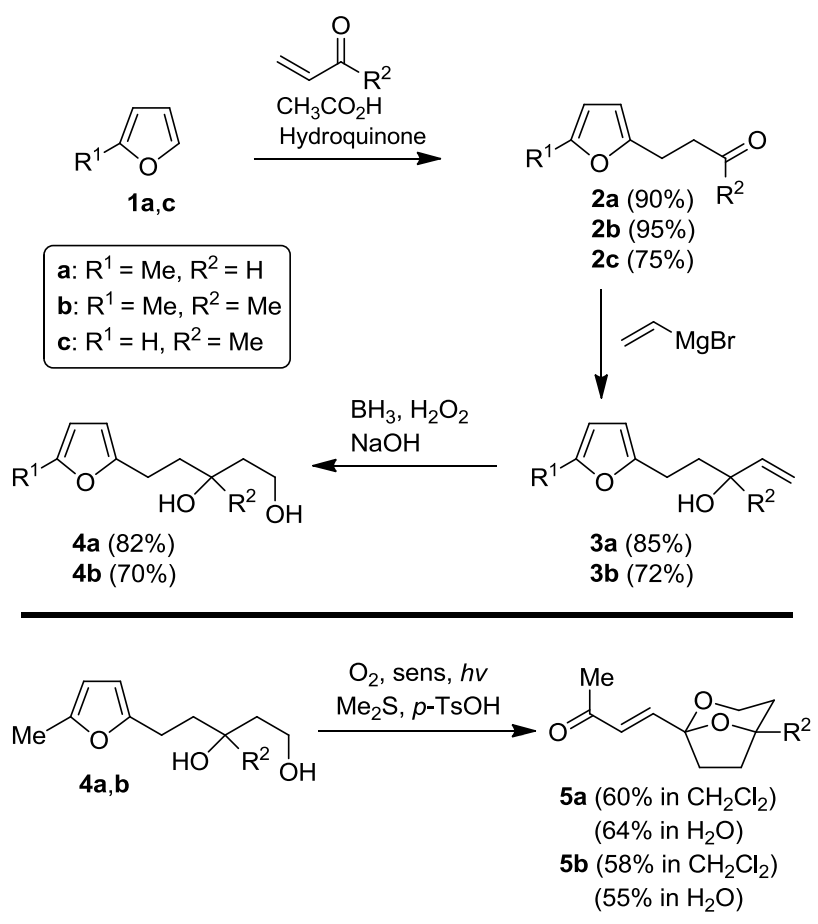

note that the intended transformation of substrate $\mathbf{A}$ into the desired product $\mathbf{F}$ is envisaged to take place as a onepot tandem reaction sequence; in other words, there will be no sequential unveiling of hydroxyl groups (no protections/deprotections), despite the threat of alternate disrupting pathways that is intrinsic when the intermediates bear multiple nucleophilic functionalities and have multiple electrophilic sites that are potentially vulnerable to attack. Based on previous experience, it was judged that the cascade reaction sequence had been finely tuned to favour the desired sequence; however, empirical evidence was keenly awaited to confirm the various assumptions that had been made.

The first test substrates $\mathbf{4 a}, \mathbf{b}$ were synthesised using a facile 3-step protocol in which 2-methylfuran (1a) was first condensed with 3-buten-2-one, or acrolein, to afford furans 2a,b in high yields (90 and $95 \%$, respectively). Addition of vinylmagnesium bromide, followed by hydroboration-oxidation, yielded the desired test substrates $\mathbf{4 a}$ and $\mathbf{4 b}$. These substrates were then subjected to standard singlet photooxidation conditions; namely, oxygen bubbling through a solution of the substrate and sensitiser (methylene blue $10^{-4} \mathrm{M}$ ), in dichloromethane at 5 ${ }^{\circ} \mathrm{C}$, whilst simultaneously being irradiated by a Variac Eimac Cermax 300W visible spectrum lamp for the short reaction time required $(2-15$ mins, monitored using tlc). All the characteristic peaks corresponding to an intermediate C-type compound (Scheme 2) were observed in crude ${ }^{1} \mathrm{H}$ NMR spectra. Excess dimethylsulphide (DMS) was then added to the solution to affect the reduction of the peroxide functionality (c.f. $\mathbf{C} \rightarrow \mathbf{D}$, Scheme 2), followed by a catalytic quantity of $p$-TsOH which it was hoped would catalyse the cyclisation rearrangement (c.f. D $\rightarrow$ F). Gratifyingly, the desired bicycles (5a and $\mathbf{b}$ ), bearing trans double bond geometry, were isolated in good yield from this cascade reaction sequence. To add to the excitement of having attained the desired products from a complex cascade reaction sequence, we also found that the procedure could be undertaken equally successfully using water as solvent (with rose bengal as photosensitiser) with all the inherent advantages this media has for improving the green credentials of this already efficient and non- polluting process. Thus, we had obtained proof for the principle that bicyclo[2.1.3] octane systems, similar to those found in the PTXs, could be efficiently synthesised in either dichloromethane or water, starting from an extremley easy-to-access furan substrate. However, to have broad synthetic scope, the newly-developed methodology would need to be extended to substrates bearing different substitution patterns, particularly those resembling other PTX family members and/or those bearing handles that would facilitate their inclusion in a full PTX synthesis.

The next strategic goal was, therefore, identified as being the inclusion of a suitable handle (in this case a $\mathrm{CH}_{2} \mathrm{OH}$ unit to be located at the equivalent of the PTXs C16 position, Scheme 1). The inclusion of an additional, unprotected primary alcohol in the photoxygenation substrate was a potentially complicating feature as it opened up the possibility of forming an alternative [5,7]bicycle instead of the desired, and, probably more thermodynamically stable, bicyclo[2.1.3] octane system (if the newly included primary alcohol were to ketalise in preference to the desired secondary alcohol). Doubt over which of these two scenaria would dominate needed to be eliminated, however unlikely the undesired appeared to be

Scheme 4. Photooxidation of 2-( $\gamma, \varepsilon, \zeta$-trihydroxyalkyl) furans

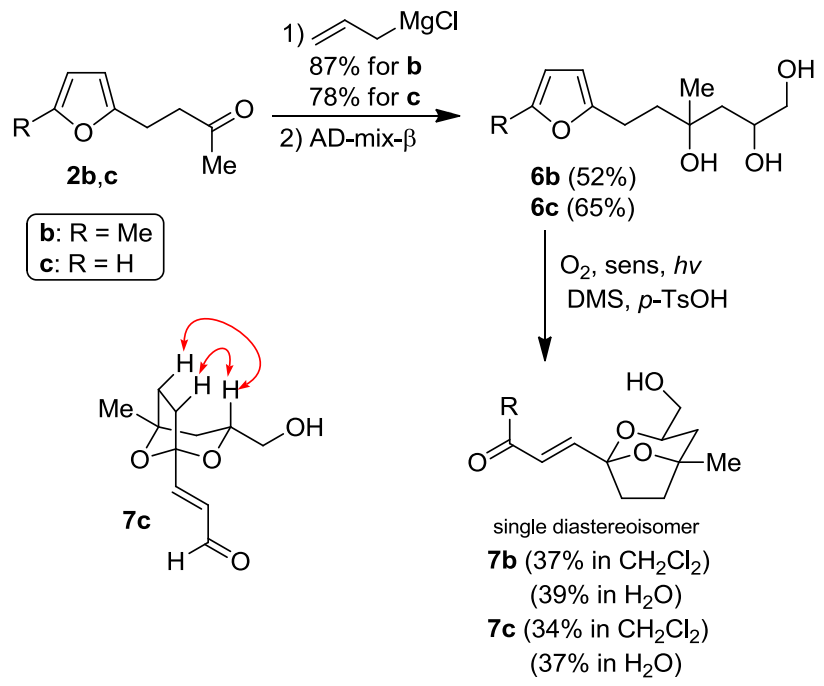


because of the reversible nature of the reactions preceeding product formation. To this end $2-(\gamma, \varepsilon, \zeta-$ trihydroxyalkyl)furans $\mathbf{6 b}$ and $\mathbf{c}$ were synthesised from the previously accessed intermediates $\mathbf{2 b}$ and $\mathbf{c}$ (Scheme 3) by the addition of allylmagnesium chloride and Sharpless asymmetric dihydroxylation of the resultant homoallylic alcohol (Scheme 4). Both $\mathbf{6 b}$ and $\mathbf{c}$ were obtained, as expected, as a 1:1 mixture of diastereoisomers. The diastereomeric mixtures were then subjected to the previously delineated singlet oxygen photooxygenation conditions (including the reduction with excess DMS and catalytic $p$-TsOH catalysed ketalisation-rearrangement steps). The desired bicycles $\mathbf{7 b}$ and $\mathbf{c}$ were both successfully isolated as single diastereoisomers. NOE studies were used to confirm that the relative stereochemistry was that of the desired PTX DE-bicycle, see Scheme 4. No starting material was recovered from this reaction and it is proposed that the absence of the undesired diastereoisomer in the final product mixture could be related to its inherent instability as was suggested by the work of Brimble ${ }^{10}$ on an analogous system. Once again the reaction could be conducted in the traditional medium of dichloromethane, or, equally, successfully, using water.

Examination of the PTX family structures shows that some form of substitution is always present at C-18 and that this substitution ranges from a simple methyl group (already modelled in our investigations) to a $-\mathrm{COOH}$ functionality and encompasses all the intermediary oxidation states (Scheme 1). Therefore, in order to develop a truly effective methodology a new variant was now targetted translating to the inclusion of a $-\mathrm{CH}_{2} \mathrm{OH}$ functionality for the "C-18" position (through which all other oxidation states, and, therefore, all PTXs, could be theoretically accessed). As in the previous examples, we aimed to include this additional and potentially complicating feature in its naked unprotected form.

To this end triol 9 was synthesised using the sequence shown in Scheme 5. The primary hydroxyl functionality of diol 4a, which had been accessed using the previously

Scheme 5. Photooxidation of furan-triol 9

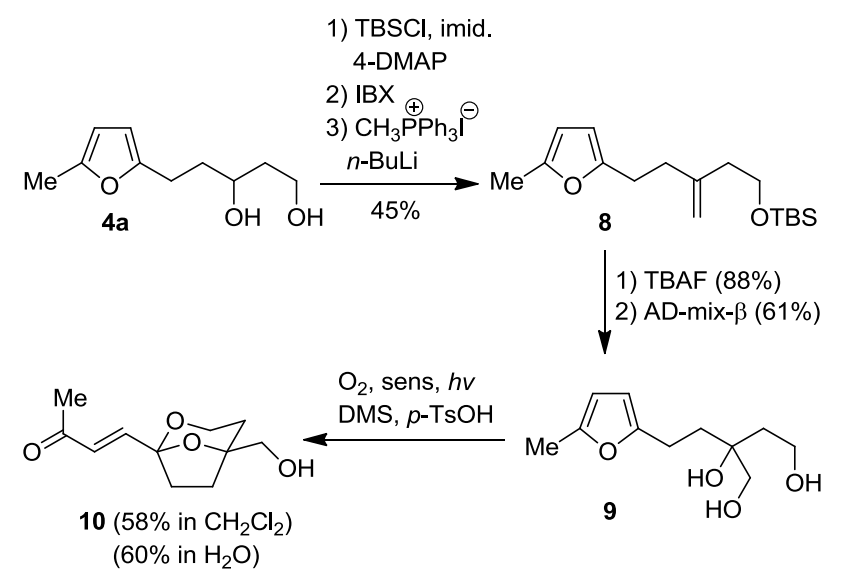

delineated chemistry (Scheme 3), was preferentially protected with a TBS group using standard conditions. The remaining secondary hydroxyl group was then oxidised using IBX and methylenation of the resultant ketone accomplished using the simple Wittig procedure to afford substrate 8. Deprotection of the primary TBS functionality using TBAF was followed by Sharpless asymmetric dihyroxylation (AD-mix- $\beta$ ) to afford triol 9. We were delighted to observe formation of the desired PTX DE-bicycle $\mathbf{1 0}$ as the sole product upon subjection of triol 9 to the previously employed photooxygenation conditions (including the DMS reduction step and the $p$ $\mathrm{TsOH}$ ketalisation rearrangement step). No products (i.e. the corresponding [6,7]-bicycle ${ }^{17}$ ) arising from the possible alternative ketalisation were observed. As before, the photooxygenation reaction could be undertaken in aqueous media. The yield for this cascade reaction sequence of $58-60 \%$ is remarkably high considering the increase in molecular complexity achieved during the course of this one-pot synthetic operation.

In summary, an extremely efficient protocol has been developed to access bicyclo[2.1.3] octane systems similar to those found in the PTX family of natural products. Primary alcohol handles which would allow for further elaboration of the fragments and variations at the "C-18" centre (also unprotected primary alcohols) were shown to be well-tolerated. The procedure involves transformation of simple and readily accessible furan substrates into the desired bicycles using a one-pot singlet oxygen-mediated cascade reaction sequence. This atom- and step-economic protocol, which utilises environmentally benign oxygen from the air as oxidant source, and, which can be undertaken in water, represents an achievement that takes us a step closer to the prized ideal green synthesis. ${ }^{1,4}$

Acknowledgment. The research leading to these results has received funding from the European Research Council under the European Union's Seventh Framework Programme (FP7/2007-2013)/ERC grant agreement no. 277588. We also thank Prof. Robert Stockman and $\mathrm{Mr}$ George Procopiou for their help in taking HRMS.

Supporting Information Available: Experimental procedures, full spectroscopic data and copies of ${ }^{1} \mathrm{H}$ and ${ }^{13} \mathrm{C}-\mathrm{NMR}$ spectra for all new compounds. This material is available free of charge via the Internet at http://pubs.acs.org.

\footnotetext{
${ }^{17}$ For formation of a similar and undesired [6,7]-bicycle in PTX work, see; ref 10 .
} 